EXTENDED REPORT

\title{
Doppler ultrasound findings in healthy wrists and finger joints before and after use of two different contrast agents
}

\author{
L Terslev, S Torp-Pedersen, N Bang, M J Koenig, M B Nielsen, H Bliddal
}

Ann Rheum Dis 2005;64:824-827. doi: 10.1136/ard.2004.028548

See end of article for authors' affiliations

Correspondence to: Dr L Terslev, Parker Institute, Department of Rheumatology,

Frederiksberg Hospital, DK 2000 Frederiksberg,

Denmark; parker@

fh.hosp.dk

Accepted

9 November 2004
Objective: To examine the effect of contrast agents on Doppler ultrasound findings in the synovial membrane in the wrist and fingers of healthy volunteers.

Material and methods: Eleven healthy subjects were included in the study 15 women and 6 men, mean age 38 years, range (20-60)). They had no clinical signs of inflammatory or degenerative joint diseases. A total of 66 joints were examined -6 joints for each subject: wrist and metacarpophalangeal (MCP) joints 1-5-before contrast injection and after Levovist and SonoVue injection with a 30 minute interval.

Results: Colour Doppler activity was detected in 10/55 (18\%) MCP joints before contrast injection and in 29/ $55(53 \%)$ and $28 / 55(51 \%)$ joints after Levovist $(p<0.0001)$ and SonoVue injection $(p=0.0001)$, respectively. A significant increase in Doppler activity in the radial $(p<0.05)$ and ulnar $(p=0.01)$ parts of the wrist joint was detected only after SonoVue injections. With spectral Doppler no difference was found in the resistive index (RI) in the vessels measured before as compared with those only detected after contrast injection.

Conclusion: The number of joints with colour Doppler activity in healthy volunteers was increased by the use of contrast agents. No changes in RI were detected. The value of contrast agents remains to be demonstrated in inflammatory diagnostics.
D oppler ultrasound is used increasingly in the evaluation of joint inflammation and has the advantage of giving an objective analysis of the disease without ionising radiation. It is accessible in many outpatient clinics, has no contraindications, and poses no problems of patient compliance. Colour and power Doppler ${ }^{1-6}$ have been used to identify the hyperaemia associated with inflammation ${ }^{1-4}$ by visualising the vascularity in the inflamed synovial membrane. With spectral Doppler it is possible to evaluate the type of flow-that is, low resistance versus high resistance, in these vessels expressed by the resistive index (RI). Previous studies have shown that low RI values are associated with inflammation. ${ }^{3-6}$

Ultrasound contrast agents in the form of microbubbles are used to enhance the scattering reflection from blood in order to increase the sensitivity of the Doppler examination. They have within the past few years been used to amplify the colour Doppler signal in patients with inflammatory joint conditions, and one study also used spectral Doppler for the examination of vessels. ${ }^{3}$ The results have been divergent. Although some studies have reported Doppler signals from healthy joints without the use of contrast agents, ${ }^{78}$ Doppler signals in healthy subjects were neither seen before nor after contrast injection in other studies. ${ }^{9-11}$ The use of contrast agents in rheumatology is still being investigated, and so far the studies have not agreed about the findings and there is no consensus about the recommendations for future use of contrast agents in inflamed joints. ${ }^{310-12}$

Previous studies have shown that it is possible to detect vascularisation in normal joints without the use of contrast agents, $^{7813}$ and this study aimed at examining the effect of contrast agents on Doppler ultrasound findings in the synovial membrane in the wrist and fingers of healthy volunteers.

\section{PATIENTS AND METHODS \\ Subjects}

Twelve healthy subjects were recruited, and 11 ( 5 women and 6 men, mean age 38 years, range $(20-60))$ were included in the study. One subject was excluded during the examination because of indisposition-probably caused by the contrast agent. The subjects were not currently engaged in heavy manual labour or performing sporting activities with their hands and had no history of arthritis, hand or wrist trauma or current symptomatology. None of the subjects had clinical signs of inflammatory or degenerative joint diseases. A total of 66 joints were examined-six joints per subject: the wrist and metacarpophalangeal (MCP) joints $1-5$.

\section{Ultrasound}

An Acuson Sequoia ultrasound system (Mountainview, California, USA) was used for the ultrasound examinations with a $15 \mathrm{MHz}$ linear array transducer for all examinations. The healthy volunteers were examined sitting in an upright position with the dominant hand placed on a cushion, fully pronated.

The dorsal side of the wrist was scanned from side to side in the longitudinal plane. In the evaluation of the wrist, the radiocarpal joint and the intercarpal joints were scanned. The wrist was evaluated at three locations: radial, central, and ulnar.

The MCP joints were scanned in the longitudinal plane in the regions that were accessible from the dorsal side: MCPI was scanned in an arch of $180^{\circ}$ from the ulnar to the radial side, MCP2 and MCP5 in an arch of $150^{\circ}$, and MCP 3-4 in an arch of $120^{\circ}$.

The colour Doppler box included the whole joint space in all joints.

\section{Colour Doppler}

Blood flow in the synovial membrane was visualised with colour Doppler. The colour Doppler settings were the same for all joints and all volunteers, with a gain setting just below the noise level using our set up for low flow-Nyquist limit $\pm 0.014 \mathrm{~m} / \mathrm{s}, 7 \mathrm{MHz}$ Doppler frequency, and lowest possible

Abbreviations: MCP, metacarpophalangeal; $\mathrm{RI}$, resistive index; $\mathrm{ROI}$, region of interest 
Table 1 Number of colour Doppler positive joints before and after contrast injection

\begin{tabular}{lccl}
\hline & $\begin{array}{l}\text { Before } \\
\text { contrast } \\
\text { injection }\end{array}$ & Levovist & SonoVue \\
Joints & 10 & $29^{* * *}$ & $28^{* * *}$ \\
\hline$M C P(n=55)$ & 4 & $7 \mathrm{NS}$ & $10^{* *}$ \\
Wrist, ulnar $(n=11)$ & 8 & $10 \mathrm{NS}$ & $10 \mathrm{NS}$ \\
Wrist, central $(n=11)$ & 2 & $1 \mathrm{NS}$ & $7^{*}$ \\
Wrist, radial $(n=11)$ & 2 & & \\
\hline NS, non-significant. & & & \\
${ }^{*} \mathrm{p} \leqslant 0.05 ;{ }^{* *} \mathrm{p} \leqslant 0.01 ;{ }^{* * *} \mathrm{p} \leqslant 0.001$. &
\end{tabular}

wall filter. These Doppler settings mostly exclude noise artefacts but include blooming artefacts. To establish that the colour pixels represented flow and not an artefact, spectral Doppler was used displaying arterial or venous flow.

\section{Spectral Doppler}

Colour Doppler was used as guidance, the spectral Doppler sampling area was placed over an intrasynovial artery, and the spectrum was obtained. The ultrasound unit traced the Doppler spectrum electronically, identifying the cardiac cycles as well as peak systolic flow and end diastolic flow. The unit then calculated the RI as (peak systolic flow-end diastolic flow)/peak systolic flow. If more than one synovial artery could be sampled, a mean RI was used. The degree of peripheral resistance is expressed numerically with the RI. ${ }^{14}$

Because the intrasynovial vessels are very small, both the artery and its concomitant veins are often sampled simultaneously even with the smallest possible Doppler gate. A flow reversal during diastole (normal in musculoskeletal tissues) will then go unnoticed because the reversed arterial flow will drown in the venous signal. To obtain uniform measurements, we therefore limited the spectral measurements to the arterial side of the Doppler line and thereby defined 1.00 as the maximum for RI.

Qualitative estimation of Doppler activity was based on the presence or absence of colour Doppler pixels in the synovium, and the effect of contrast agents was determined as the change in the number of joints with visible flow. Quantitative estimation of vascularisation in the synovial membrane was estimated by the colour fraction and the RI. The colour fraction was determined in those joints that showed Doppler activity both before and after contrast injection in order to estimate the effect of contrast agents in these joints. The colour Doppler image with maximum colour activity was selected. The digitally stored colour Doppler image in DICOM format was transferred to a processing program (a $\beta$ version of DataPro; Noesis, Courtaboeuf, France). The synovial membrane was traced, indicating the region of interest (ROI). The number of colour pixels and the total number of pixels in the ROI were counted ${ }^{3}$ and the number of colour pixels was then expressed in relation to the total number of pixels in the same ROI.

The RI was measured, if possible, in all joints displaying a colour Doppler signal before contrast injection. After injection with contrast agent, the RI was measured, if possible, in the new joints displaying flow in order to investigate the flow profile in previously invisible vessels.

\section{Contrast injection}

In this study two different echo enhancing contrast agents were used: Levovist (Schering, Berlin, Germany) and SonoVue (Bracco, Milano, Italy). This was done in order to compare our findings with previous studies using Levovist. Because Levovist is no longer in production we chose SonoVue for future comparison. Levovist is a preparation of

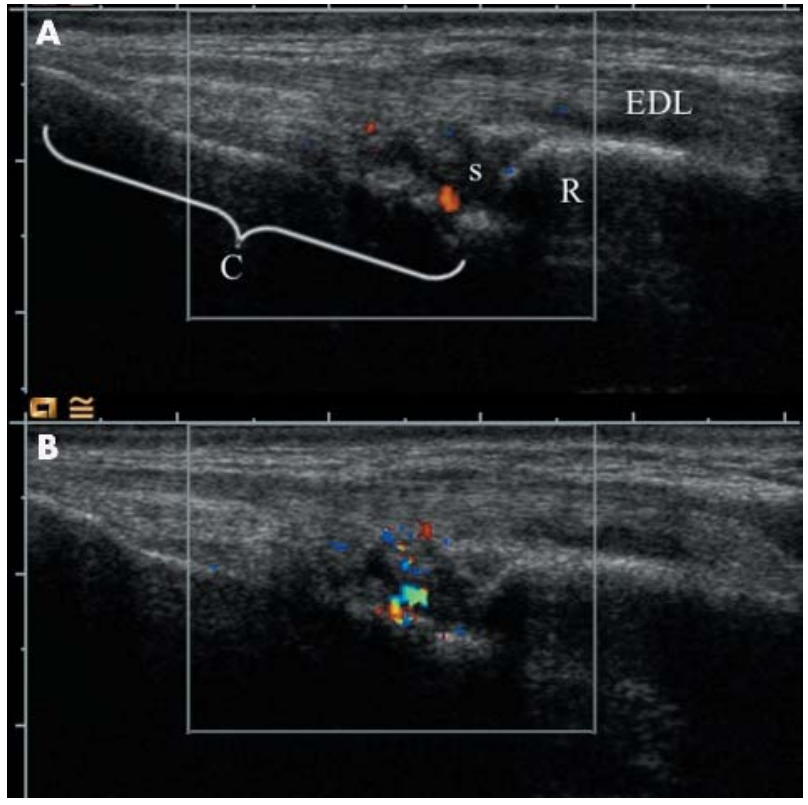

Figure 1 Colour Doppler in the wrist before and after SonoVue injection. The images are longitudinal through the extensor digitorum longus tendon (EDL). The surface of the radius (R) and carpal bones (C) are seen as bright reflectors. The synovium of the radiocarpal joint(s) is seen as a anechoic/hypoechoic mass with extensions that are synovial duplications. (A) Before contrast injection. A single Doppler focus is visible inside the synovium. (B) After contrast injection. A larger Doppler focus is visible.

air filled microbubbles stabilised by palmitic acid in a galactose based suspension. SonoVue is a suspension of sulphur hexafluoride microbubbles stabilised by a phospholipidic monolayer in $0.9 \%$ saline.

First a bolus of $7 \mathrm{ml}$ Levovist $(300 \mathrm{mg} / \mathrm{ml})$ was injected through an 18 gauge catheter into a cubital vein of the contralateral arm followed by a $20 \mathrm{ml}$ saline bolus injection. The measurements were repeated on wrist and MCP joints immediately after observing a substantial increase in blooming artefact in the radial artery as a sign of the contrast effect. Thirty minutes later $2.4 \mathrm{ml}$ SonoVue was injected, followed by a $20 \mathrm{ml}$ saline bolus injection, and the measurements were repeated again with the joints in the same order.

Examination of each patient lasted about 5 minutes. In all the patients an enhanced Doppler signal was still present at the end of the examination.

\section{Statistics}

Statistical analysis was performed comparing paired groups of categorical data in a hypothesis test. A Wilcoxon signed rank sum test was used to evaluate the changes in the colour fraction. For the differences in mean values in the RI before and after contrast injection an independent sample $t$ test was used. The level of significance was chosen at $p \leqslant 0.05$.

\section{RESULTS}

Table 1 shows the Doppler ultrasound findings for the individual joints.

Colour Doppler activity was detected in 10/55 (18\%) MCP joints before contrast injection and in 29/55 (53\%) joints after Levovist $(p<0.0001)$ and in $28 / 55(51 \%)$ after SonoVue injection $(p=0.0001)$ (table 1$)$. When the wrist was investigated at three different locations owing to the composite structure of this joint, no significant effect after Levovist injections was seen. After SonoVue injections, however, Doppler activity in the radial $(\mathrm{p}<0.05)$ and in the 
ulnar part $(p=0.01)$ of the joint increased significantly. However, when the wrist was looked upon as one joint, there was no significant effect in the use of a contrast agent as 10 of 11 wrists had Doppler activity before injection and 11 of 11 after both Levovist and SonoVue injections.

When the joints with colour Doppler activity both before and after contrast injection were investigated, there was an increase in the mean colour fraction from 0.04 before contrast injection to 0.06 after Levovist and 0.09 after SonoVue (fig 1). This increase was not statistically significant. For both contrast agents one MCP joint in two different patients showed colour Doppler activity before and not after contrast injection.

When the three different parts in the wrist joint (ulnar, radial, and central part) were looked at separately, one ulnar and two radial parts in three different patients showed colour Doppler activity before and not after Levovist injection. A similar phenomenon was not seen after SonoVue injection.

The mean (SD) RI for the vessel found before contrast injection (13 joints) was $0.89(0.04)$ and the mean RI in the vessels only detected after SonoVue injection ( 23 joints) was 0.88 (0.04), range $0.25-1.00$. There was no statistically significant difference between the groups.

\section{Adverse events}

In one subject a reaction with nausea occurred immediately after the injection of SonoVue. The episode lasted about 3 hours and the subject was excluded from further ultrasound examination. The subject recovered without sequelae.

\section{DISCUSSION}

The present study demonstrated the presence of Doppler activity before contrast injection in 10/55 MCP joints and in $10 / 11$ wrist joints of healthy volunteers with no history of arthritis, hand or wrist trauma, or current symptomatology, which is higher than our previous findings. ${ }^{8}$ The contrast agents increased the colour Doppler activity significantly in the MCP joints and in the wrist joint if this was investigated as three separate compartments, displaying a higher number of visible vessels. An increase in colour fraction was also found in the joints with already existing flow before contrast injection, indicating either an increase in the number of visible vessels or an increase in the blooming artefact as also described in previous studies. ${ }^{10}$

No statistically significant difference was found in the RI in the vessels seen before contrast injection and those made visible after SonoVue injection. In other words, with a high end Doppler the flow profile in normal joints was the same for visualised and non-visualised vessels-visualisation in the latter enabled by contrast in this study. When RI is used to categorise inflammation it seems appropriate to assume a normal value for RI when no measurable vessels are visible. Some normal vessels both in this study and in a previous study $^{8}$ had RI values $<0.8$ and we regard these as outliers and expect normal resting musculoskeletal tissue to have values in the range above 0.8 and most often close to 1.00. Similar investigations of this phenomenon remain to be performed in arthritic joints, which lose their detectable Doppler activity in response to treatment. With contrast, it would presumably be possible to detect and measure "non-visualised" vessels previously having Doppler activity in the untreated state. It is possible that these vessels will have a normal RI and thus behave just like the non-visualised vessels in this normal material.

The findings in this study differ from those of previous studies, which did not demonstrate Doppler signals using contrast agents in healthy joints. ${ }^{10}{ }^{11}$ A reasonable explanation for these divergent observations is a variation in the quality of the equipment with differences in Doppler sensitivity. With lower Doppler sensitivity fewer vessels will be visible as opposed to the present results obtained with a high end Doppler, which displayed vessels even in normal resting joints.

Our findings support the observation in most of the studies evaluating inflamed joints in the hand, ${ }^{3911}$ with an increase in the number of Doppler positive joints as well as the number of colour pixels after contrast injection. However, in one report an increase in the number of Doppler positive joints in patients with rheumatoid arthritis was not found. ${ }^{10}$ As suggested by the authors, the most likely explanation for this divergence was that none of the patients had low inflammatory activity.

The present findings as well as previously reported findings may be explained by the following model: all healthy and inflamed joints have vessels with varying degrees of flowhigh perfusion in vessels supplying an inflamed region, or low perfusion in vessels supplying non-inflamed regions. The sensitivity of the Doppler then defines how many vessels may be detected (fig 2A). A high end Doppler will detect both low and high perfusion vessels, whereas a low end Doppler will only detect vessels with high perfusion. When injecting the contrast agent, the signal from all vessels is enhanced, thereby increasing the number of visible vessels. A low end Doppler may only detect more of the inflamed vessels,
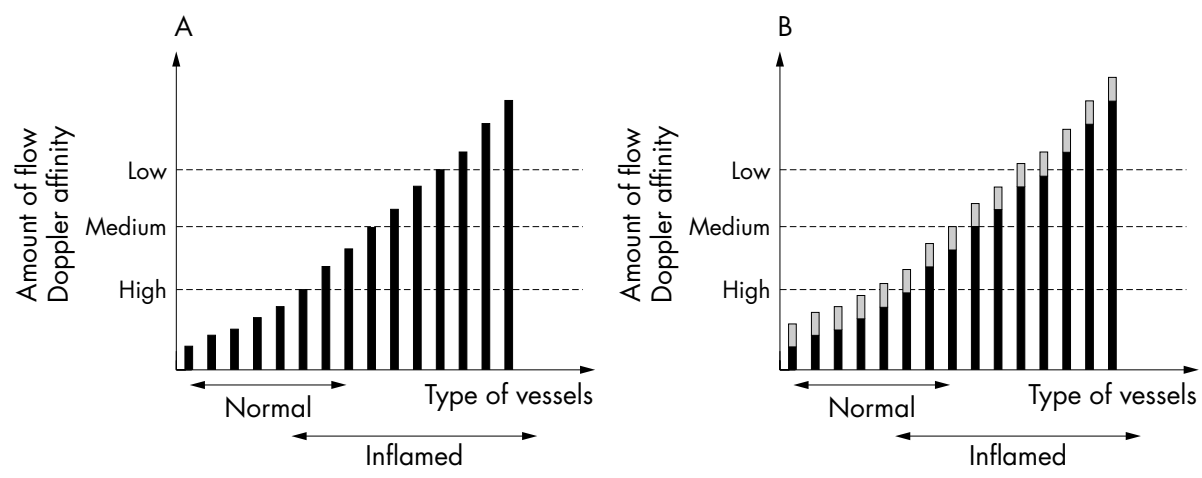

Figure 2 Hypothetical model for the interaction between Doppler ultrasound and amount of synovial flow. (A) The synovial vessels are ranked on the $x$ axis with increasing ability to be detected by Doppler to the right. An overlap between vessels of the normal synovium and inflamed synovium is expected. Thresholds for detection of flow for low, medium, and high sensitivity Doppler are shown with dashed lines. In this model only high sensitivity Doppler will detect flow in a normal synovium and then will detect only a few vessels. (B) After contrast injection, all vessels have an increased ability to be detected by Doppler. The bars have increased in height and some vessels have crossed the Doppler thresholds. According to the model no Doppler activity will be found in normal joints before or after contrast injection if a medium sensitivity Doppler is used. Also, if a contrast study on patients with rheumatoid arthritis does not include mildly inflamed joints, then contrast injection will not result in additional joints becoming Doppler positive. 
whereas a high end Doppler may also detect an increase in the number of normal vessels (fig 2B).

This raises the question of whether the vessels that are only seen after contrast injection provide additional information. The vessels visualised in normal resting joints after contrast injection are, of course, not pathological. In parallel, we speculate that additional vessels visualised after contrast injection in inflamed joints need not be part of the inflammatory process; they may well be vessels with normal perfusion in non-inflamed parts of the synovium. Based on the present evidence we cannot agree that the use of contrast agents provides essential information in inflamed joints. ${ }^{911}$ Furthermore, the results must depend on the quality of the Doppler imaging. With relatively insensitive Doppler equipment we agree that vessels which become visible with contrast agents may form part of the inflammation and additional information thereby obtained. Continuous use of contrast agents will, however, generate substantial costs, which seem better used for upgrade of equipment - thereby conserving the ultrasound examination as non-invasive.

The use of contrast agents is very similar to enhancing the sensitivity of the Doppler. At present, with high end equipment we see flow in many normal joints and with contrast we see even more. Continuing improvement of the Doppler sensitivity may very well in the near future-even without contrast-match the results found in this study with contrast. The possibility of adverse events after injecting contrast underlines the demand for maintaining the noninvasiveness of the ultrasound procedures.

Our ability to detect flow is not a fully developed technique and the diagnosis of arthritis will in some institutions rely on the mere presence of colour and in others on the presence of more than normal colour. Cut off levels defining thresholds between normal and pathological Doppler activity must therefore be defined. These cut off levels must be individually set depending on the quality of the equipment, the settings used, and whether contrast is used or not. Early arthritis is probably the most difficult to diagnose because the synovial membrane may be barely increased in thickness and the perfusion may be only slightly increased in these joints compared with normal. The diagnosis of early arthritis based on ultrasound findings necessitates a definition of normal flow-and, as a consequence, recognition of a slightly increased flow also.

\section{CONCLUSION}

The number of joints (wrists and MCP joints) with colour Doppler activity found in healthy volunteers was increased by the use of contrast agents. The Doppler sensitivity in newer high end ultrasound machines allows detection of normal synovial flow even without the use of contrast agents. The RI did not change after contrast. The value of contrast agents remains to be demonstrated in inflammatory diagnostics.

\section{ACKNOWLEDGEMENTS}

This study was supported by the Oak Foundation.

\section{Authors' affiliations}

L Terslev, S Torp-Pedersen, M J Koenig, H Bliddal, Parker Institute, Department of Rheumatology, Frederiksberg Hospital, DK 2000 Frederiksberg, Denmark

N Bang, M B Nielsen, Department of Radiology, Ultrasound Unit X 4123, Rigshospitalet, DK $2100 \varnothing$, Denmark

The study was approved by the local ethical committee and informed consent was obtained from each participant.

\section{REFERENCES}

1 Schmidt WA, Volker L, Zacher J, Schlafke M, Ruhnke M, Gromnica-Ihle E. Colour Doppler ultrasonography to detect pannus in knee joint synovitis. Clin Exp Rheumatol 2000; 18:439-44.

2 Walther M, Harms H, Krenn V, Radke S, Faehndrich TP, Gohlke F. Correlation of power Doppler sonography with vascularity of the synovial tissue of the knee joint in patients with osteoarthritis and rheumatoid arthritis. Arthritis Rheum 2001;44:331-8.

3 Qvistgaard E, Rogind H, Torp-Pedersen S, Terslev L, Danneskiold-Samsoe B, Bliddal H. Quantitative ultrasonography in rheumatoid arthritis: evaluation of inflammation by Doppler technique. Ann Rheum Dis 2001;60:690-3.

4 Terslev L, Torp-Pedersen S, Qvistgaard E, Danneskiold-Samsoe B, Bliddal H. Estimation of inflammation by Doppler ultrasound: quantitative changes after intra-articular treatment in rheumatoid arthritis. Ann Rheum Dis 2003;62:1049-53.

5 Troltzsch M. [Color Doppler study in patients with rheumatoid arthritis and scleroderma. ] Z Rheumatol, 1994;53:2-6.

6 Midiri M, lovane A, Finazzo M, Brancatelli G, Gallo C, Lagalla R. [Color Doppler-echo in rheumatoid arthritis with extra-articular location. Preliminary experience. ] Radiol Med (Torino), 1999:98:123-6.

7 Hau M, Schultz H, Tony HP, Keberle M, Jahns R, Haerten R, et al. Evaluation of pannus and vascularization of the metacarpophalangeal and proximal interphalangeal joints in rheumatoid arthritis by high-resolution ultrasound (multidimensional linear array). Arthritis Rheum 1999:42:2303-8.

8 Terslev L, Torp-Pedersen S, Qvistgaard E, Von der Recke P, Bliddal H. Doppler ultrasound findings in healthy wrists and finger joints. Ann Rheum Dis 2004;63:644-8.

9 Magarelli N, Guglielmi G, Di Matteo L, Tartaro A, Mattei PA, Bonomo L. Diagnostic utility of an echo-contrast agent in patients with synovitis using power Doppler ultrasound: a preliminary study with comparison to contrastpower Doppler ultrasound: a preliminary study

10 Szkudlarek M, Court-Payen M, Strandberg C, Klarlund M, Klausen T, Ostergaard M. Contrast-enhanced power Doppler ultrasonography of the metacarpophalangeal joints in rheumatoid arthritis. Eur Radiol 2003; 13:163-8.

11 Klauser A, Frauscher F, Schirmer M, Halpern E, Pallwein L, Herold M, et al. The value of contrast-enhanced color Doppler ultrasound in the detection of vascularization of finger joints in patients with rheumatoid arthritis. Arthritis Rheum 2002;46:647-53.

12 Carotti M, Salaffi F, Manganelli P, Salera D, Simonetti B, Grassi W. Power Doppler sonography in the assessment of synovial tissue of the knee joint in rheumatoid arthritis: a preliminary experience. Ann Rheum Dis 2002;61:877-82.

13 Torp-Pedersen T, Torp-Pedersen S, Bliddal H. Diagnostic value of ultrasonography in epiconylitis. Ann Intern Med 2002;136:781-2.

14 Pourcelot L. L'examen Doppler des vaisseaux périphériques. Paris: AC-D Production, 1982 